\title{
Theoretical Deduction of the Hubble Law Beginning with a MoND Theory in Context of the $\Lambda$ FRW-Cosmology
}

\author{
Nelson Falcon, Andrés Aguirre \\ Laboratory of Physics of the Atmosphere and the Outer Space, University of Carabobo, Valencia, Venezuela \\ Email: nelsonfalconv@gmail.com, aaguirre3@uc.edu.ve
}

Received 11 September 2014; revised 8 October 2014; accepted 3 November 2014

Academic Editor: Luigi Maxmilian Caligiuri, University of Calabria, Italy

Copyright (C) 2014 by authors and Scientific Research Publishing Inc.

This work is licensed under the Creative Commons Attribution International License (CC BY). http://creativecommons.org/licenses/by/4.0/

c) (i) Open Access

\section{Abstract}

We deduced the Hubble law and the age of the Universe, through the introduction of the Inverse Yukawa Field (IYF), as a non-local additive complement of the Newtonian gravitation (Modified Newtonian Dynamics). As a result, we connected the dynamics of astronomical objects at great scale with the Friedmann-Robertson-Walker $\Lambda F R W$ ) model. From the corresponding formalism, the Hubble law can be expressed as $v=(4 \pi[G] / c) r$, which was derived by evaluating the IYF force at distances much greater than $50 \mathrm{Mpc}$, giving a maximum value for the expansion rate of the universe of $\mathrm{H}_{0}^{(\max )} \simeq 86.31 \mathrm{~km} \cdot \mathrm{s}^{-1} \cdot \mathrm{Mpc}^{-1}$, consistent with the observational data of 392 astronomical objects from NASA/IPAC Extragalactic Database (NED). This additional field (IYF) provides a simple interpretation of dark energy as the action of baryonic matter at large scales. Additionally, we calculated the age of the universe as $11 \mathrm{Gyr}$, in agreement with recent measurements of the age of the white dwarfs in the solar neighborhood.

\section{Keywords}

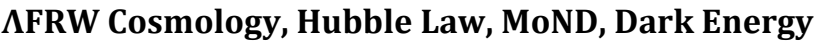

\section{Introduction}

The idea of a model for a universe in continuous and constant expansion emerged from the pioneering work of Hubble, Slipher and Humason [1]. This dynamic description of the universe began with early studies on relati- 
vistic cosmology [2] and is the foundation of the Big Bang theory, which explicitly uses the so-called Hubble law.

Hubble law was empirically proposed by Hubble [1], who noted a roughly linear relation between velocities and distances among nebulae, and saw that the relation appears to dominate the distribution of velocities. The mathematical expression for this relation proposed by Hubble, so-called Hubblelaw, is usually written as

$$
v=H_{0} r
$$

where $v$ (in units of $\mathrm{km} \cdot \mathrm{s}^{-1}$ ) is the recessional velocity of a given astronomical object, whose distance from the Earth, $r$, is measured in Mpc, and $H_{0}$ is the Hubble constant (in $\mathrm{km} \cdot \mathrm{s}^{-1} \cdot \mathrm{Mpc}^{-1}$, that can be alternatively written as $H_{0}=100 \mathrm{hm} \cdot \mathrm{s}^{-1} \cdot \mathrm{Mpc}^{-1}$, where $h$ is the dimensionless Hubble parameter and takes values between 0 and 1.

Since the discovery of the accelerating expansion of the universe through the study of high red shift supernovae [3] [4], the current cosmological model uses the Hubble law together with Friedmann equations as the basis of the Standard Model of Big Bang cosmology. Friedmann equations constitute the solutions of the Einstein field equations of the General Theory of Relativity for the Friedmann-Robertson-Walker (FRW) metric under the additional assumption of the isotropic and homogeneous universe at large scales (Cosmological Principle).

Although Hubble law represents the first observational test of the expansion of the universe, and today supports the actual cosmological model [5], it has not been theoretically deduced, so many hypotheses have arisen to this end, and even more new theories have emerged as alternative for the velocity-distance law. Among the alternatives is Browne [6], who determined that Hubble law is a linear approximation of a more general exponential law, but it was conceived for a deSitter universe with no matter. Segal et al. [7] by studying IRAS data proposed asquare law, as given by Lundmark, however Strauss \& Koranyi [8] reviewed Segal's research and studied IRAS data too and determined that observations actually support Hubble law, the same result was obtained for galaxies from CfA and ESO/LV [9]. Pascual-Sánchez [10] determined a generalized Hubble law which introduces two additional terms to the usual Hubble law produced by the angular expansion, but this conception implies an anisotropic universe in conflicts with the Cosmological Principle. At this point, Hubble law has remained unalterable, and therefore the latest theories seem to look for deriving the velocity-distance law as was proposed by Hubble, namely, they look for a theoretical deduction of the Hubble law. Liu [11] derived the Hubble law under a hypothesis that eliminates the need for dark energy, nevertheless he used a non-conventional form of the FRW metric with the time defined as relative to some hypothetical time where the line element was or will be the Minkowskian, which has not been found by observations. Sorrell [12] proposed that Hubble law, as result of an expanding universe, is really a working hypothesis, instead he considered the hypothesis proposed by Zwicky of the tired-light, but nowadays it is well known that this theory is not supported by observations, in fact it does not explain theanisotropies in the CMB. Recently, Sanejouand [13] opted for a non-standard form of the Hubble law, assuming a new definition of the red shift based infrequencies rather than wavelength, establishing a new paradigm for the spectroscopy.

One of the biggest problems in the Big Bang cosmology, closely linked to the expansion of the Universe and the Hubble law, is the evidence of the accelerated expansion of the Universe, commonly referred as dark energy, whose understanding is still far from complete. Also, the inconsistency between the observed average density of matter and the density required for flatness of the universe, a problem known as the missing mass, has become the paradigm of the hypothetical non-baryonic dark matter. This discrepancy between the astronomical observations of the density of matter and expected in $\Lambda \mathrm{FRW}$ model in the Big Bang theory, has prevailed in the last years. An alternative to the paradigm of non-baryonic dark matter is the theory of Modified Newtonian Dynamics (MoND), which involve changes in the Newton's law of gravitation (inverse square law).

In this sense, one possibility to solve both problems: dark matter and dark energy, is the non-local gravitation recently proposed by Falcón [14], which basically is a MoND theory. According to which the force of gravitation would be the result of two fields generated by the ordinary baryonic matter, a first term as Newton law of the inverse square and an additional long-range term.

The inclusion of this second term in the force of gravity, consistent with Eötvös-like experiments, can reconcile the $\Lambda$ FRW model with observables of the Big Bang, without the paradigm of non-baryonic dark matter. Additionally, gives an explanation for dark energy, and allows us to theoretically deduce the Hubble law.

In this paper, we will show that the Hubble law can be derived from the MoND theory proposed by Falcón in a natural way through the corresponding condition of cosmological scales. To this end, in Section 2 we will re- 
view the paper of Falcón emphasizing the repulsive behavior of the non-local gravitational field at large scales, giving a starting point for deducting the Hubble law. The theoretical deduction of the Hubble law and even an analytical determination of the Hubble constant will be given in Section 3. In Section 4, we will contrast the determined Hubble constant with the observational data of 392 objects selected from the NASA/IPAC Extragalactic Database (NED), also a brief discussion about the cosmic age problem is given. Finally, the conclusions are given in Section 5.

\section{MoND with Non-Local Gravitational Term}

Current Big Bang cosmology assumes Newtonian gravitation as the only fundamental force at astronomical scales, giving a complete determination of the dynamics of the universe. However, from this idea we face serious difficulties to describe the behavior of the Universe: 1) galaxy rotation curves are not explained without the inclusion of non-baryonic dark matter, whose fundamental nature and properties are completely unknown; 2) into the rich galaxy clusters, the observed mass of stars and the gas mass inferred from the X-ray diffuse emission is significantly less than that required to hold these systems gravitationally stable; and 3) the accelerated expansion of the universe violates our understanding about how gravity works at cosmological scales (see [14] for details).

The simplest way for modeling the accelerated cosmic expansion is by introducing a cosmological constant into the Einstein's field equations so it can represent a hypothetical negative pressure of the vacuum of space, also called dark energy. However this is given as a disconnected idea from the dynamics of the astronomical objects, which is limited to the Newton's law of gravitation.

While Newtonian gravitation (inverse square law) has been highly supported by laboratory experiments and satellites, there is no experimental evidence to confirm its validity beyond the Solar System [15]. That is why it has raised the Modified Newtonian Dynamics (MoND) theories such as proposed by Milgrow [16] that solves the galaxy rotation problem originating from non-baryonic dark matter. Following this line, Falcón [14] [17] proposed a modification of the Newtonian gravitation by adding a non-local term that contains Milgrow's theory as a particular case and establishes a possible connection for the dynamics at large scale and FRW formalism. This additional term was constructed by the specular reflection of the potential of Yukawa, so that we decided to named it: Inverse Yukawa Field (IYF). This interaction is given by the baryonic matter (as the Newtonian gravity), and shows a null contribution at scale of the Solar System $\left(\sim 10^{-4} \mathrm{pc}\right)$, in agreement with measurements on Earth, weakly attractive at interstellar distances $(\sim 10 \mathrm{kpc})$, consistent with MoND theory (as a solution of the galaxy rotation problem), strongly attractive at scales of galaxy clusters $(\sim 1 \mathrm{Mpc})$, in accordance with Abell radius, and repulsive at cosmological scales $(\gg 50 \mathrm{Mpc}$ ), in agreement with the expansion of the universe (see Figure 1). This interaction has a potential per unit of mass of the form

$$
U(r) \equiv U_{0}\left(r-r_{0}\right) \mathrm{e}^{-\alpha / r}
$$

where $U_{0}=U_{0}(M)$ is the magnitude of the potential (in units of $\mathrm{N} \cdot \mathrm{kg}^{-1}$ ) as a function of the baryonic matter, $\alpha \sim 2.5 h^{-1} \mathrm{Mpc}$ and $r_{0} \sim 50 h^{-1} \mathrm{Mpc}$ are constants.

Then, the proposed modification considers the contribution of both the Newtonian and the non-local gravitational field, so that the dynamics at all scales is determined by the force per unit of mass as

$$
F(r)=G \frac{M}{r^{2}}-\frac{U_{0}(M)}{r^{2}} \mathrm{e}^{-\alpha / r}\left[r^{2}+\alpha\left(r-r_{0}\right)\right]
$$

where it is important to note that there is a dependence on the baryonic matter only.

In particular, a zero contribution of the non-local term can be verified at distances below $10^{-4} \mathrm{pc}$, in agreement with measurements on Earth as Eötvös-like experiments. However, a measurable contribution can be observed at $45 \mathrm{AU}$, indeed the IYF provides a sunward acceleration of the order of $10^{-11} \mathrm{~m} \cdot \mathrm{s}^{-2}$ consistent with acceleration presented by the pioneer spacecraft [18]. On the other hand, at scales of tens of kiloparsec, the Newtonian contribution can be neglected and the IYF term shows a MoND-like behavior of the form

$$
F\left(r \ll r_{0}\right) \simeq\left(\frac{U_{0}(M) r_{0}}{2}\right) \frac{1}{r}
$$

solving the galaxy rotation problem. Also, the non-local IYF, evaluated in the Abell radius ( $r \sim 1.2 \mathrm{Mpc})$, pro- 
vides an additional force, two hundred and fifty times greater than the Newton's force, so it could solve the missing mass problem in galaxy clusters first identified by Zwicky.

From Figure 1, it is clear that IYF potential gives a constant repulsive force at cosmological scales $(\gg 50 \mathrm{Mpc})$ as

$$
F(r \gg 50 \mathrm{Mpc}) \simeq U_{0}(M)
$$

providing an asymptotic cosmic acceleration, consistent with the observations. This opens the possibility to describe the behavior of the cosmological constant by setting it as a dynamical term with the form $\Lambda \equiv \Lambda(r) \propto U(r)$, giving a link between the dynamics of astronomical objects, due the Newtonian and IYF force, with Friedmann equations, which are only modified by the introduction of the dynamism of $\Lambda$ as

$$
\begin{aligned}
& \left(\frac{\dot{R}(t)}{R(t)}\right)^{2}+\frac{k c^{2}}{R^{2}(t)}=\frac{8 \pi G}{3} \rho+\frac{\Lambda(r) c^{2}}{3} \\
& 2 \frac{\ddot{R}(t)}{R(t)}+\left(\frac{\dot{R}(t)}{R(t)}\right)^{2}+\frac{k c^{2}}{R^{2}(t)}=-\frac{8 \pi G}{c^{2}} P+\Lambda(r) c^{2}
\end{aligned}
$$

where the dot denotes the time derivate of the scale factor $R(t), k=-1,0,+1$ is the scalar curvature for a open, flat and closed universe respectively, $c$ is the speed of light, $G$ is the gravitational constant, $\rho$ is the total mass-energy density, and $P$ the pressure.

The introduction of the non-zero contribution of the cosmological constant brings a modification to the usual form of the matter density parameter, $\Omega_{m}$, in terms of the energy of the IYF, $\Omega_{\mathrm{IYF}}$. Then, Equation (6) is now

$$
\frac{k c^{2}}{R^{2}(t)}=H_{0}^{2}\left[\Omega_{m}\left(1+\Omega_{\mathrm{IYF}}\right)+\Omega_{\Lambda}-1\right]
$$

where the dark energy density parameter (or cosmological density parameter), $\Omega_{\Lambda}$, is defined as usual. Hence, the flatness condition $(k=0)$ is fulfilled by Friedmann equation without the assumption of non-baryonic dark matter.

For a complete interpretation of the behavior of the IYF potential and details about the cosmological consequences by adding the IYF to Newtonian dynamics and to FRW cosmology see [14]. Finally, the repulsive behavior of this non-local term provides an starting point for studying the dynamics at large scale, and therefore for deducting theoretically the Hubble law.

\section{Theoretical Deduction of Hubble Law}

A numerical value for $U_{0}$ can be found by studying the gravitational Poisson equation, noting that the IYF potential must satisfy this equation.

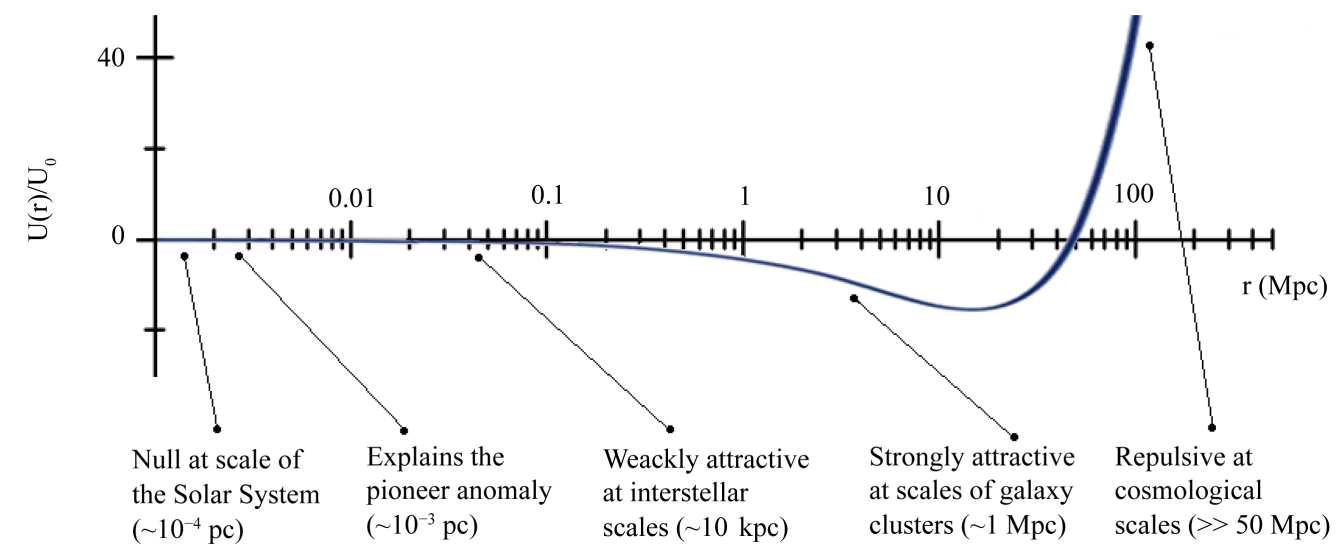

Figure 1. IYF potential per unit of mass as function of the distance between objects gravitationally bounded (see [14] for details). 
Usually, the Poisson equation is written for the Newtonian gravitational case as $\nabla^{2} \Phi_{N}=4 \pi G \rho$, however since the introduction of the new interaction we must add a scalar field corresponding to the IYF, so $\nabla^{2} \Phi_{N} \rightarrow \nabla^{2} \Phi_{N}+\nabla^{2} \Phi_{\mathrm{IYF}}$, but because we are evaluating the asymptotic limit of cosmological scales, the Newtonian contribution is not important. Thus, the Poisson equation with IYF for a spatial matter distribution, $\rho(r)$, is

$$
\nabla^{2} U(r)=4 \pi G \rho(r)
$$

with $U(r)$ given by Equation (2). Therefore, calculating the Laplancian operator with spherical symmetry, and observing that the resulting function and the density are linearly dependent for $r \gg 50 \mathrm{Mpc}$ (in a mathematical sense), we have that the constants $U_{0}$ and $4 \pi G$ must satisfy the equality $U_{0}=4 \pi G$, where the units are specified as

$$
U_{0}=4 \pi G \times 1 \mathrm{~kg} \cdot \mathrm{m}^{-2} \simeq 8.39 \times 10^{-10} \mathrm{~N} \cdot \mathrm{kg}^{-1}
$$

and the same for the matter density

$$
\rho(r)=\frac{\mathrm{e}^{-\alpha / r}}{r^{4}}\left[2 r^{2}(r+\alpha)+\alpha^{2}\left(r-r_{0}\right)\right] \times 1 \mathrm{~kg} \cdot \mathrm{m}^{-2}
$$

taking into account that this equality works for $r$ 's much greater than $50 \mathrm{Mpc}$, so that the Newtonian contribution is null.

Here, we note that the obtained magnitude, $U_{0}$, for the IYF potential gives a maximum value, as result of evaluating the behavior of the baryonic matter density in the asymptotic case of cosmological scales.

On the other hand, in Section 2 we saw that the IYF, as anon-local term, shows a repulsive behavior at cosmological scales ( $\gg 50 \mathrm{Mpc}$ ), providing an asymptotic cosmic acceleration in accordance with accelerated expansion of the universe. This allows atheoretical deduction of the Hubble law, as a lineal proportionality between recessional velocities and distances.

Consider a particle (galaxy, galaxy cluster, nebulae, etc.) with nonzero rest mass under the influence of the IYF force. The contribution of the Newtonian gravitational force is not important at cosmological scales (i.e. at $1 \mathrm{Mpc}, \quad F_{\text {Newton }} \propto r^{-2} \sim 10^{-49} \mathrm{~N}$ at least). Thus, the equation of motion is only given by the force per unit of mass of the IYF. Additionally, since the IYF is conservative, we can write

$$
\frac{\mathrm{d}^{2} r}{\mathrm{~d} t^{2}}=\frac{\mathrm{d} U}{\mathrm{~d} r}=\frac{U_{0}(M)}{r^{2}} \mathrm{e}^{-\alpha / r}\left[r^{2}+\alpha\left(r-r_{0}\right)\right]
$$

where $U_{0}=U_{0}(M)$ depending of the baryonic matter, $M$, that causes the field.

Then, it is possible to obtain an expression of the velocity by integrating Equation (12) as follow $v=\int\left(\mathrm{d}^{2} r / \mathrm{d} t^{2}\right) \mathrm{d} t$. Here, the time interval $\mathrm{d} t$ is measured through the photons giving the recessional velocity of the particle. Therefore,

$$
v=\int \frac{\mathrm{d} U}{\mathrm{~d} r} \frac{\mathrm{d} r}{c} \Rightarrow v=\frac{1}{c} U+\text { const }
$$

With $v$ as the time derivate of the comoving distance, $r$. Then, the velocity is proportional to the IYF potential. Actually, because the Hubble flow is observed at cosmological distances, we should evaluate the IYF potential at $r \gg 50 \mathrm{Mpc}$, so that $r_{0} / r \simeq 0$ and $\mathrm{e}^{-\alpha / r} \simeq 1$. Hence, from Equation (2), we obtain

$$
v=\frac{U_{0}}{c} r
$$

where without loss of generality we assumed the initial condition $v=0$ at $r=0$, resulting in a null integration constant. Here, from analogy with the Hubble law we find the Hubble constant as $U_{0} / c$, where the magnitude of the IYF potential is given by Equation (10). Even more, because we are evaluating the asymptotic limit of cosmological scales in the IYF force, we can determine the limit value of this proportionality constant as

$$
H_{0}^{(\max )}=\frac{4 \pi G}{c} \times 1 \mathrm{~kg} \cdot \mathrm{m}^{-2} \simeq 86.31 \mathrm{~km} \cdot \mathrm{s}^{-1} \cdot \mathrm{Mpc}^{-1}
$$

so that the Hubble law can be written as 


$$
v=H_{0}^{(\max )} r
$$

Note that Equation (16) basically is equal to Equation (1), establishing a linear relation between recessional velocities and distances for a given particle (galaxy, cluster of galaxy, nebulae, etc.), just under the assumption of cosmological scales, in agreement with the current cosmological model. Additionally, the limit value of the linearly constant gives the maximum expansion rate of the universe, again as product ofstudy distances much greater than $50 \mathrm{Mpc}$.

In the next section, we will test the $H_{0}^{(\max )}$ value with the observational data from NED, under criteria that allow studying the velocity-distance relation at large scale.

\section{Observational Test and Discussions}

Although the first determination of the Hubble constant was $H_{0}=500 \mathrm{~km} \cdot \mathrm{s}^{-1} \cdot \mathrm{Mpc}^{-1}$ [1], today it is well known that this proportionality constant takes values less than $100 \mathrm{~km} \cdot \mathrm{s}^{-1} \cdot \mathrm{Mpc}^{-1}$. In fact, Sandage [19] gave the first reasonable estimated of the Hubble constant by studying Cepheids, he obtained that $H_{0}$ is about $75 \mathrm{~km} \cdot \mathrm{s}^{-1} \cdot \mathrm{Mpc}^{-1}$. Four decades later, Freedman et al. [20] studied objects over the range of about $60-400$ Mpc, using Cepheids, and determined $H_{0}=72 \pm 8 \mathrm{~km} \cdot \mathrm{s}^{-1} \cdot \mathrm{Mpc}^{-1}$. Bonamente et al. [21] studied galaxies with redshift between 0.14 and 0.89 , obtaining that the Hubble constant is $77.6_{-12.5}^{+14.9} \mathrm{~km} \cdot \mathrm{s}^{-1} \cdot \mathrm{Mpc}^{-1}$. After nine years of recordingand analysis of the CMB data from WMAP, Bennett et al. [22] calculated that $H_{0}=69.32 \pm 0.80$ $\mathrm{km} \cdot \mathrm{s}^{-1} \cdot \mathrm{Mpc}^{-1}$. The latest value of the Hubble constant was determined by Ade et al. [23], who studied the CMB through Planck satellite, where the data fitted $H_{0}=67.15 \pm 1.20 \mathrm{~km} \cdot \mathrm{s}^{-1} \cdot \mathrm{Mpc}^{-1}$, being the value accepted today.

In this section, we will contrast our value for the Hubble constant, of $H_{0}^{(\max )} \simeq 86.31 \mathrm{~km} \cdot \mathrm{s}^{-1} \cdot \mathrm{Mpc}^{-1}$, with the observational data provided by the NASA/IPAC Extragalactic Database (NED).

In order to verify the Hubble law and our value for the Hubble constant, we will use the primitive technique used by Hubble, which consists in plotting the observational measurements of the velocity (via red shift) and the distance of a set of objects such as galaxies, quasars, radio sources, X-ray sources, infrared sources, etc. For this end, we considered the Master List of Redshift-Independent Extragalactic Distances of 15339 galaxies provided by NED (Version 9.2.0). The observational measurements were filtered by: 1) recent measurements (year of publication from 2009); 2) distantobjects ( $r \gg 50 \mathrm{Mpc})$; 3) red shift from 0.0167 to 0.33 ; and 4) accurate measurements (with maximum error of $0.5 \%$ ). As a result, the list was reduced to392 objects (the complete list of the 392 objects can be found on https://db.tt/vwlVdhVM). Here, we must clarify that in order to filter errors by peculiar motions we used red shifts above 0.0167 , so errors are under $6 \%$ [20], and due the theoretical assumption of $v=c z$ we set red shifts below 0.33 , so that Lorentz factor is equally under $6 \%$ and the relativistic effects are neglected.

A Hubble diagram for the 392 galaxies, in a range of 50 - $1400 \mathrm{Mpc}$, is shown in Figure 2. Through a linear fit we found that $H_{0} \simeq 83.56 \pm 0.59 \mathrm{~km} \cdot \mathrm{s}^{-1} \cdot \mathrm{Mpc}^{-1}$ (solid line), so that the determined maximum expansion rate of $H_{0}^{(\max )}=86.31 \mathrm{~km} \cdot \mathrm{s}^{-1} \cdot \mathrm{Mpc}^{-1}$ disagrees about $3 \%$ only. Nevertheless, because our prediction works at the limit of cosmological scales, we would hope that observational measurements show an asymptotic behavior to an expansion rate of $86.31 \mathrm{~km} \cdot \mathrm{s}^{-1} \cdot \mathrm{Mpc}^{-1}$ at distances even greater than $1500 \mathrm{Mpc}$. Actually, in Figure 2, we can note that observational data slightly suggest an upper slope for distances above $1000 \mathrm{Mpc}$.

In Figure 2, we additionally note that the data suggest a lower slope for distances lower than 500 Mpc. Actually, a linear fit at that scales gives a Hubble constant of $H_{0}=72.14 \pm 2.47 \mathrm{~km} \cdot \mathrm{s}^{-1} \cdot \mathrm{Mpc}^{-1}$ (dashed line), which agrees with the value given by Freedman et al. of $H_{0}=72 \pm 8 \mathrm{~km} \cdot \mathrm{s}^{-1} \cdot \mathrm{Mpc}^{-1}$ (via Cepheid variablesapplied over the range of about $60-400 \mathrm{Mpc}$ ) and Blakeslee et al. [24] of $H_{0}=72 \pm 15 \mathrm{~km} \cdot \mathrm{s}^{-1} \cdot \mathrm{Mpc}^{-1}$ (via SBF with range of applicability until $125 \mathrm{Mpc}$ ). Clearly, from the used method this consistence is expected.

On the other hand, an additional result can be obtained through the determined Hubble constant: the age of the universe, $\tau$, which can be calculated as

$$
\tau=H_{0}^{-1} \int_{0}^{\infty}\left[(1+z)^{3} \Omega_{m}\left(1+\Omega_{\mathrm{IYF}}\right)+\Omega_{\Lambda}\right]^{-1 / 2} \frac{\mathrm{d} z}{1+z}
$$

[14], where $\Omega_{m}$ is the matter density parameter but only including baryonic matter, $\Omega_{\mathrm{IYF}}$ is a density parameter emerged from the contribution of the IYF, $\Omega_{\Lambda}$ is the cosmological density parameter, and $z$ is the usual red shift. Here, we note that Equation (17) is reduced to the conventional form, in the $\Lambda F R W$ model with $k=0$, 


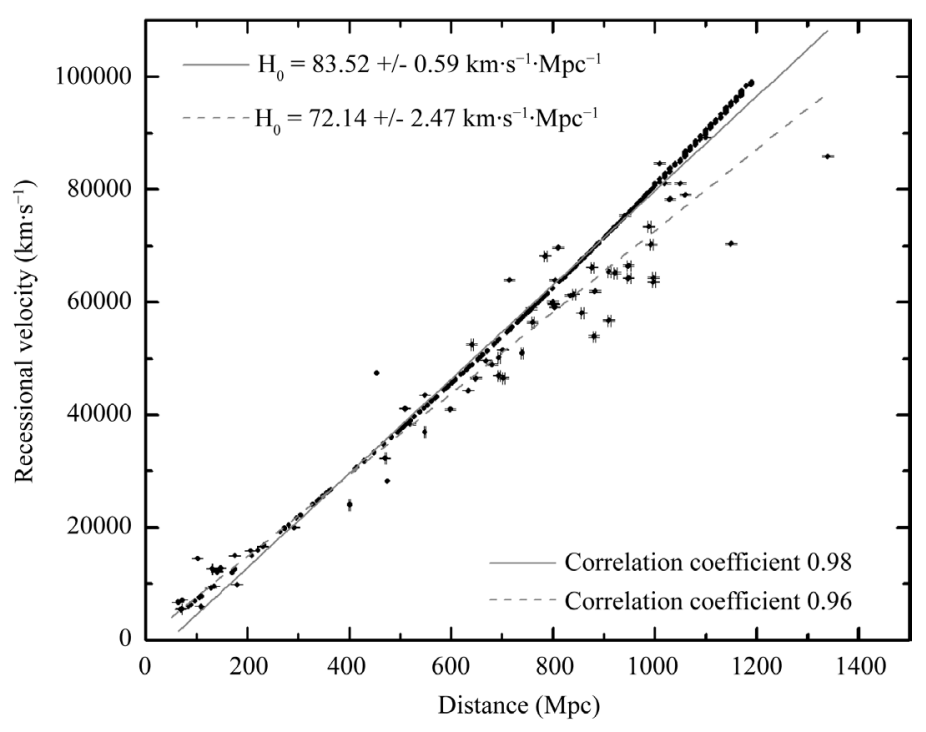

Figure 2. Hubble diagram for objects at 50 - 1400 Mpc (data from NED).

when the IYF is zero.

Numerical integration of Equation (17), for $H_{0}^{(\max )}=86.31 \mathrm{~km} \cdot \mathrm{s}^{-1} \cdot \mathrm{Mpc}^{-1}, \Omega_{m}=0.03$ [25], $\Omega_{\mathrm{IYF}}=8.80$ and $\Omega_{\Lambda}=0.71$ [14], gives an age of the universe of $\tau \simeq 11 \mathrm{Gyr}$, in agreement with the age of the white dwarfs in the solar neighborhood [26] and, from the Copernican Principle, with the age of the universe. Here, we must say that there exist astronomical objects with ages greater than $11 \mathrm{Gyr}$, i.e., B495 is $14.54 \mathrm{Gyr}, \mathrm{B} 024$ is 15.25 Gyr and B050 is $16.00 \mathrm{Gyr}$ [27], however the determination of the age of the oldest globular clusters, via HR diagram, introduces intrinsic errors of about $25 \%$, in which case they would be consistent with an age of 11 Gyr.

\section{Conclusions}

The inclusion of a long-range component in the law of gravitation allows linking the Hubble law with the dynamics of the large-scale Universe. Particularly, if the non-locality of gravitation is included through apotential as shown here, Yukawa Inverse type, we can connect the dark energy with cosmological constant and derive from there the Hubble law, consistent with the formalism of the Big Bang, and astronomical observations, without resorting to the paradigm of non-baryonic dark matter, or an "exoticphysics".

The inclusion of a long-range component in the law of gravitation, through an inverse potential Yukawa-like, represents the collective contribution of the gravitational effects of large-scale, on the order of tens of megaparsec caused by ordinary baryonic matter. In this sense, the IYF explicitly includes the Mach principle in the formalism of FRW cosmology, as Einstein pretended with the Theory of General Relativity.

The prescription of the Hubble constant in terms of the fundamental constants, as in Equation (15), appears to correspond to the observational data for distant objects, whose distance and red shift are independently known; as we can see in Figure 2. Note that the Hubble constant is not measured directly by the WMAP and Planck satellites, but rather its value is inferred from the power spectrum of the cosmic background radiation (CMB) together with other cosmological variables through multiple statistics correlation, or maximum likelihood.

For the nearest objects, with distances less than a hundred megaparsec, the Hubble constant would seem less than true value, because in these ranges, the contribution of the IYF field is less, as was shown in Figure 1.

A current Hubble constant $\left(H_{0}\right)$ of higher value, such as $H_{0} \simeq 83.56 \pm 0.59 \mathrm{~km} \cdot \mathrm{s}^{-1} \cdot \mathrm{Mpc}^{-1}$, implies a more recent age for the universe, but still, this value is surprisingly similar to that inferred for the age of the oldest white dwarfs in the Milky Way. Obviously, the Milky Way would have to be as old as the universe itself under the Copernican Principle, which is the very foundation of the Big Bang theory.

\section{Acknowledgements}

This research has made use of the NASA/IPAC Extragalactic Database (NED) which is operated by the Jet 
Propulsion Laboratory, California Institute of Technology, under contract with the National Aeronautics and Space Administration.

\section{References}

[1] Hubble, E. (1929) A Relation between Distance and Radial Velocity among Extra-Galactic Nebulae. Proceedings of the National Academy of Sciences, 15, 168-173. http://dx.doi.org/10.1073/pnas.15.3.168

[2] Lemaître, G. (1927) A Homogeneous Universe of Constant Mass and Increasing Radius Accounting for the Radial Velocity of Extra-Galactic Nebulae. Annales de la Société Scientifique de Bruxelles, 47, 49-59.

[3] Riess, A. G., et al.(1998) Observational Evidence from Supernovae for an Accelerating Universe and a Cosmological Constant. The Astronomical Journal, 116, 1009-1038. http://dx.doi.org/10.1086/300499

[4] Perlmutter, S., et al. (1999) Measurements of $\Omega$ and $\Lambda$ from 42 High-Redshift Supernovae. The Astrophysical Journal, 517, 565-586. http://dx.doi.org/10.1086/307221

[5] Freedman, W. and Madore, B. (2010) The Hubble Constant. Annual Review of Astronomy and Astrophysics, 48, 673710. http://dx.doi.org/10.1146/annurev-astro-082708-101829

[6] Browne, P.F. (1962) The Case for an Exponential Redshift Law. Nature, 193, 1019-1021. http://dx.doi.org/10.1038/1931019a0

[7] Segal, I.E., Nicoll, J.F., Wu, P. and Zhou, Z. (1993) Statistically Efficient Testing of the Hubble and Lundmark Laws on IRAS Galaxy Samples. The Astrophysical Journal, 411, 465-484. http://dx.doi.org/10.1086/172849

[8] Strauss, M. and Koranyi, D. (1993) Tests of the Hubble Law from the Luminosity Function of IRAS Galaxies. arXiv:astro-ph/9308028

[9] Choloniewski, J. (1995) New Test for the Hubblelaw. arXiv:astro-ph/9504035

[10] Pascual-Sánchez, J.-F. (2000) A Generalized Linear Hubble Law for an Inhomogeneous Barotropic Universe. arXiv:gr-qc/0010076

[11] Liu, J.M. (2005) Modified Hubble Law, the Time-Varying Hubble Parameter and the Problem of Dark Energy. arXiv:physics/0507018 [physics.gen-ph]

[12] Sorrell, W.H. (2009) Misconceptions about the Hubble Recession Law. Astrophysics and Space Science, 323, $205-211$. http://dx.doi.org/10.1007/s10509-009-0057-z

[13] Sanejouand, Y.H. (2014) A Simple Hubble-Like Lawin Lieu of Dark Energy. arXiv:1401.2919 [astro-ph.CO]

[14] Falcón, N. (2013) Modification of the Newtonian Dynamics in ${ }_{\Lambda}$ FRW-Cosmology an Alternative Approach to Dark Matter and Dark Energy. Journal of Modern Physics, 4, 10-18. http://dx.doi.org/10.4236/jmp.2013.48A003

[15] Gundlach, J.H. (2005) Laboratory Tests of Gravity. New Journal of Physics, 7, 205. http://dx.doi.org/10.1088/1367-2630/7/1/205

[16] Milgrom, M. (1983) A Modification of the Newtonian Dynamics: Implications for Galaxies. The Astrophysical Journal, 270, 371-389. http://dx.doi.org/10.1086/161131

[17] Falcón, N. (2011) MoND with Einstein’s Cosmological Term as Alternative to Dark Matter. Revista Mexicana de Astronomía y Astrofísica (Serie de Conferencias), 40, 11-12.

[18] Turyshev, S.G. and Toth, V.T. (2010) The Pioneer Anomaly. Living Reviews in Relativity, 13, 4. http://dx.doi.org/10.12942/lrr-2010-4

[19] Sandage, A. (1958) Currents Problems in the Extragalactic Distance Scale. The Astrophysical Journal, 127, 513-526. http://dx.doi.org/10.1086/146483

[20] Freedman, W., Madore, B.F., Gibson, B.K., Ferrarese, L., Kelson, D.D., Sakai, S., et al. (2001) Final Results from the Hubble Space Telescope Key Project to Measure the Hubble Constant. The Astrophysical Journal, 553, 47-72. http://dx.doi.org/10.1086/320638

[21] Bonamente, M., Joy, M.K., LaRoque, S.J., Carlstrom, J.E., Reese, E.D. and Dawson, K.S. (2006) Determination of the Cosmic Distance Scale from Sunyaev-Zel'dovich Effect and Chandra X-Ray Measurements of High-Redshift Galaxy Clusters. The Astrophysical Journal, 647, 25-54. http://dx.doi.org/10.1086/505291

[22] Bennett, C., Larson, D., Weiland, J.L., Jarosik, N., Hinshaw, G., Odegard, N., et al. (2012) Nine-Year Wilkinson Microwave Anisotropy Probe (WMAP) Observations: Final Maps and Results. The Astrophysical Journal Supplement Series, 208, 20. http://dx.doi.org/10.1088/0067-0049/208/2/20

[23] Ade, P.A.R., Aghanim, N., Alves, M.I.R., Armitage-Caplan, C., Arnaud, M., Ashdown, M., et al. (2013) Planck 2013 Results. I. Overview of Products and Scientific Results. arXiv:1303.5062 [astro-ph.CO]

[24] Blakeslee, J., Lucey, J., Barris, B., Hudson, M. and Tonry, J. (2001) A Synthesis of Data from Fundamental Plane and 
Surface Brightness Fluctuation Surveys. Monthly Notices of the Royal Astronomical Society, 327, 1004-1020. http://dx.doi.org/10.1046/j.1365-8711.2001.04800.x

[25] Freedman, W. and Turner, M. (2003) Colloquium: Measuring and Understanding the Universe. Review of Modern Physics, 75, 1433-1447. http://dx.doi.org/10.1103/RevModPhys.75.1433

[26] Tremblay, P.E., Kalirai, J.S., Soderblom, D.R., Cignoni, M. and Cummings, J. (2014) White Dwarf Cosmochronology in the Solar Neighborhood. arXiv:1406.5173 [astro-ph.SR]

[27] Wang, S., Li, X. and Li, M. (2010) Revisit of Cosmic Age Problem. Physical Review D, 82, 103006.

http://dx.doi.org/10.1103/PhysRevD.82.103006 
Scientific Research Publishing (SCIRP) is one of the largest Open Access journal publishers. It is currently publishing more than 200 open access, online, peer-reviewed journals covering a wide range of academic disciplines. SCIRP serves the worldwide academic communities and contributes to the progress and application of science with its publication.

Other selected journals from SCIRP are listed as below. Submit your manuscript to us via either submit@scirp.org or Online Submission Portal.
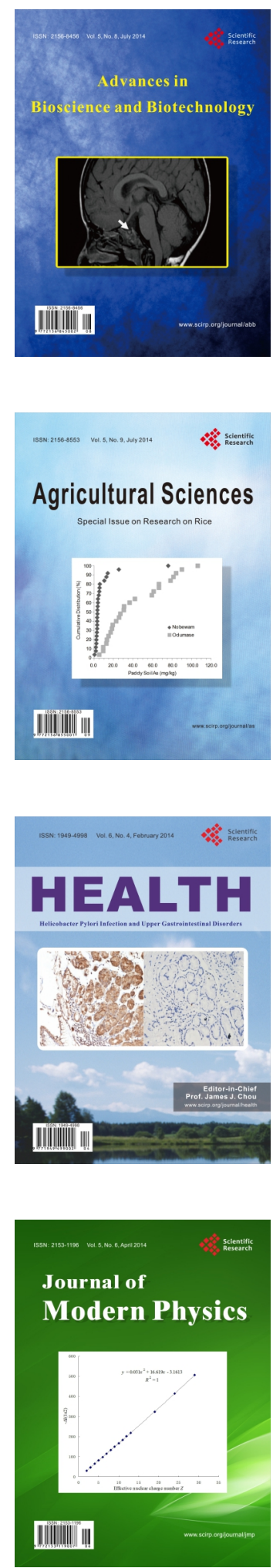
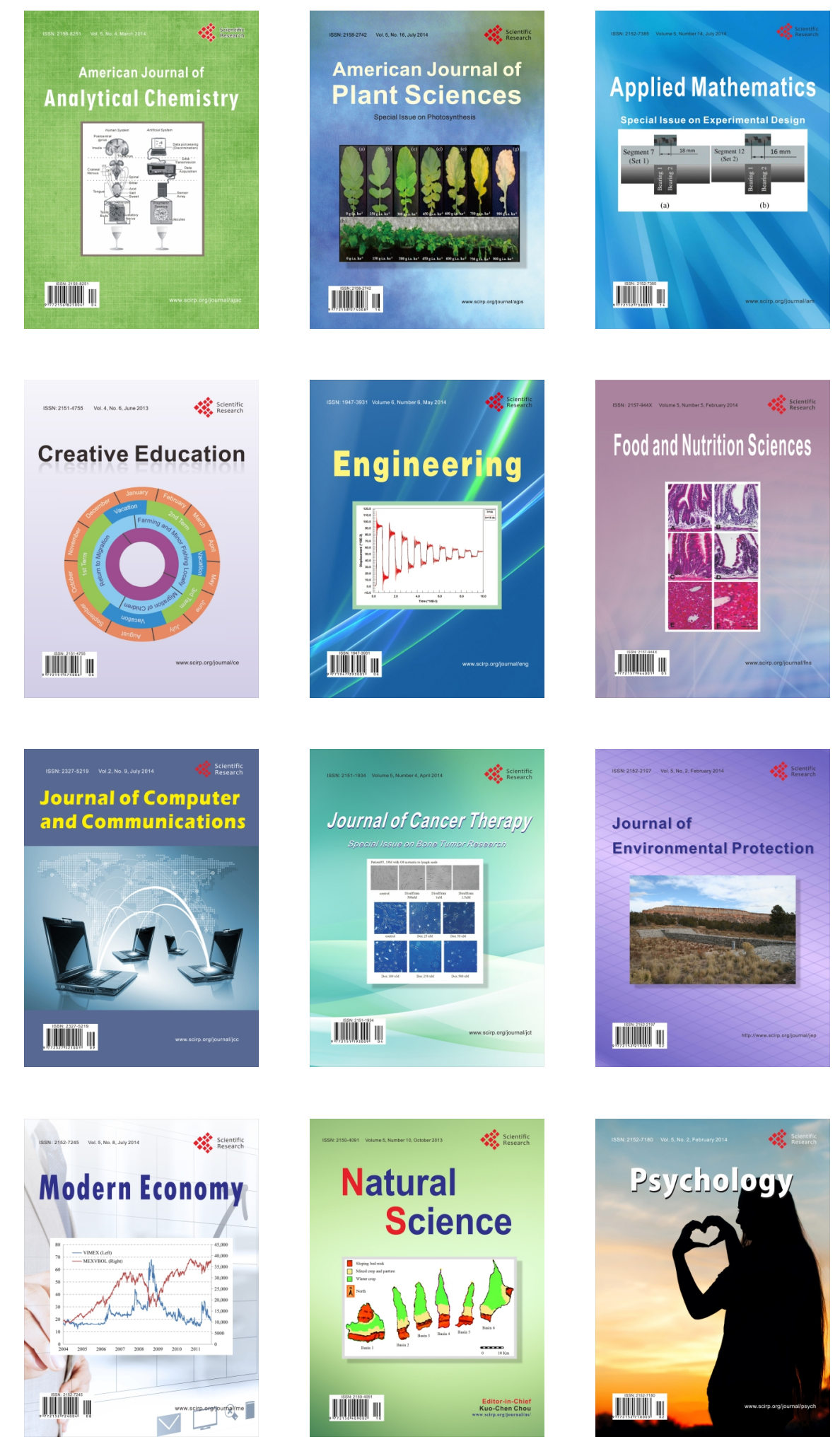